\title{
Research on Design of DC Motor Control System Based on microcontroller
}

\author{
Zhouying $^{1, \text { a }}$ \\ ${ }^{1}$ Chongqing College of Electronic Engineering, Chongqing 401331, China \\ azhouying_cq@163.com
}

Keywords: Microcontroller, DC motors, Control System

\begin{abstract}
DC motors convert electrical energy into mechanical energy, compared with AC motors, DC motors have many advantages good speed performance, high starting torque and overload, etc., it is widely used in machinery industry. MCU control DC motor is a typical application in mechanical and electrical control, just between the two ends of the DC motor control coupled with a voltage difference between the voltage it will rotate, changing the applied voltage across can change the direction of rotation. This paper designs DC motor control system based on microcontroller is presented. We use STC89C52 micro controller as kernel controller, TA7267BP as driver chip, and four keys to control the DC motor reversions, break corresponding state indicators. Hardware circuit and achievable method of software are also presented choice in controlling micro DC motor stop, and light the system is a better.
\end{abstract}

\section{Theoretical Introduction of DC Motor}

DC motor has been widely used in various fields among which single-phase, two-phase, three equally endless variety of operating mode, and each class of brushless DC motor drive system is divided into multiple, rather half-bridge drivers and full bridge drivers become the most widely used drive. Full-bridge driving mode can be divided into a variety of the most commonly used is the star and angled connector, select the drive mode will directly affect the performance and cost of the motor, so select the appropriate drive way is a top priority.

DC simple internal structure, conducive to the maintenance of the motor, and the complete elimination of the common motor by a mechanical brush and commutator caused by failure, but also greatly increases the life of the motor. Work efficiency has been significantly improved due to the use of permanent magnet brushless DC motor generates a constant, continuous magnetic field, so it is currently the most efficient motor in an electrical machine, in most conditions its permanent magnet having a relatively magnetic coefficient ${ }^{[1]}$.

Periphery Interface Chip microcontroller series is produced by American Microchip products. PIC microcontroller hardware system with its unique design and instruction, and gradually adopted the majority of engineering staff. Microchip is a set of development, development and production as one of professional single chip manufacturer, its product integrated application system design ideas, with strong technical characteristics. Products using the new pipeline structure, single-byte instruction system, embedded Flash, and 10 bit A / D converter. So that it has excellent performance, single-chip development represents a new trend. PIC MCU with high, medium and low grades three, to meet the development needs of different users, suitable for application in various fields.

\section{Hardware Design of Control System}

Microcontroller biggest feature is not to engage in purely functional accumulation, but from reality, attention to product performance and price ratio, by developing a variety of models to meet the different levels of application requirements. In practical terms, the different application requirements for Microcontroller functions and resources are different. PIC MCU using the basis of Princeton and Harvard architecture with the unique architecture of the Harvard bus architecture, the chip completely internal data bus and instruction bus separation for different byte-wide, effective 
extension instruction word length to lay the technical basis ${ }^{[2]}$. Fig.1 shows the hardware circuit structure of systems.

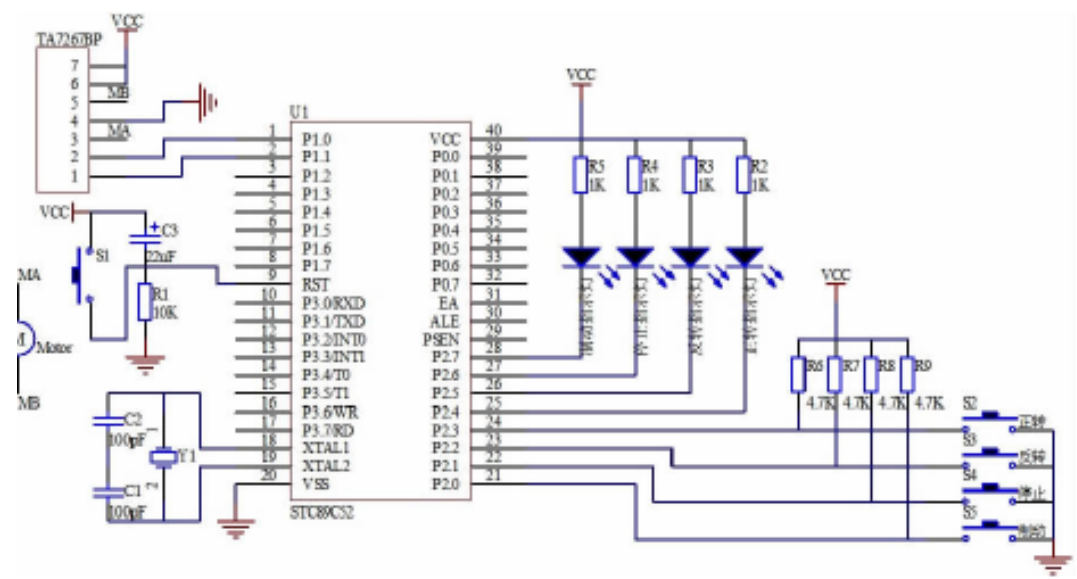

Fig. 1.The hardware circuit structure of systems

P1.0 and P1.1 are connected with the driver IC TA7267BP 2,1 pin Microcontroller by changing the level of the two pins, to achieve the four states of the motor control. (2) P2.0 and P2.3 are connected with four control buttons to control the motor forward, reverse, stop, brake. (3) P2.4 a P2. 7, respectively, and four status lights connected to the display for the respective operating state of the motor. As ASIC chip MC33035 DC motors speed control system, which can be completed IC chip output rotor position detection signal and the PWM signal at the same time. Although the structure of the system can be simplified, reducing system cost, but also limits the further improvement of the system function, resulting in most places we can't use.

A digital signal processor (DSP) can programmatically system function improvements and has good computing power, it can take advantage of their internal structure complex system control algorithms. It is possible to achieve both motor speed control system to adjust the speed and current regulation loop digital control loop, so it can be more intuitive and effective brushless DC motor speed control system to regulate all-digital mode. This can be determined by the position of the rotor sensor value obtained. Speed reference circuits by STC89C52 microcontroller A / D converter input port as the speed of a given circuit. First, the current detection circuit can detect a current signal, a weak current output voltage signal of a certain relationship, after the signal is amplified by the amplifier to the controller A / D sampling part ${ }^{[3]}$.

\section{Software Overall Program of Control System}

Designer uses modular design, functional requirements functional requirements of the program modules division binding system, the system comprising: detecting rotational speed and rotor position calculation; measurement of the motor phase currents. Microcontroller based DC motor control system, with the following features: with 8-bit A / D converter input, high drive current, I / $\mathrm{O}$ pins can directly drive digital (LED) display for each I / O pin maximum sink current 25mA; each I / 0 pins pull maximum current 25mA, two-way programmable independently pin, 16-bit timer / counter TMRI, sleep still counting. Built-in power-on reset circuit ((POR), Power-timers to ensure the establishment of a stable operating voltage oscillation timer to ensure the stability of the oscillation build, power reset the lock, that is, when the chip supply voltage drops to the set value after time, to keep the chip is reset when the power supply voltage returns to normal recovery operation, program security bit, can anti-illegal copy of the program code, power-down protection circuit ${ }^{[4]}$.

Subroutine modules and interrupt service routines included in the main program mainly finishes the following functions initialize the system for setting and enable the microcontroller associated pin; detecting the host machine control panel whether to issue the motor start command; after receiving the start command by PI algorithm slowly increasing the duty cycle PWM signal output port of the motor slow start; detecting whether the host machine control panel to issue 
commands to adjust the motor speed to ensure the motor to follow a given speed and stable operation of the interrupt service routine feature is satisfied when an interrupt trigger condition. We stop the current code execution jumps to the interrupt service routine, the interrupt service routine is executed, jump to the original position to stop the code execution to continue. Interrupt service routine has a good response speed throughout the code, in order not to affect the execution of other code, and an interrupt service routine should not be too long.

In the closed-loop control system, the output of the system through the input terminal detecting device introduced into the system, as the input signal and a feedback system compares the calculated deviation signal input and feedback signal. The error signal produced by the regulator controls on the controlled object, correct the deviation, so the deviation gradually reduced, and finally to the input and output reached nearly unlimited. Closed-loop control system to improve the immunity system, improve the control precision of the system, widely used in motor control systems.

\section{The Detection of Software Runs}

Software runs in the simulation environment through input source, compile, revise, single stepping, breakpoints, execution, execution at full speed program execution process was observed to result in the specified location, the logical structure of the software debugging correctly. Next, debug panel display module, only the right display to be able to clear or not properly follow the debugging process. Secondly, debug analog to digital conversion module. When adjusting analog to digital conversion routines to debug one by one for each channel. First debug channel 0: The channel number is set to 0 , and 0 to the inlet channel connected to voltage source, a voltage between the $0-5 \mathrm{~V}^{[5]}$. Fig.2 shows the control panel of system.

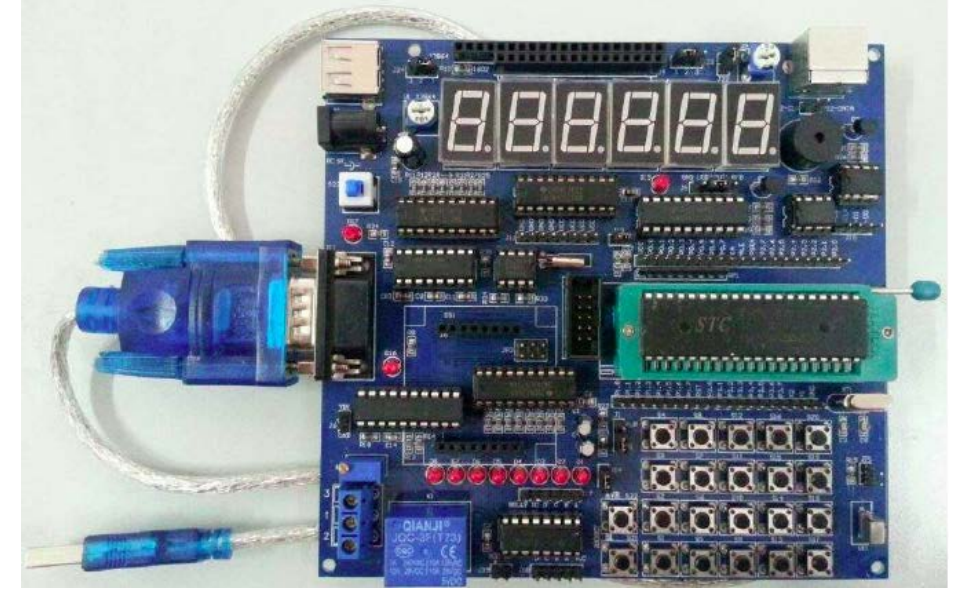

Fig. 2 .The control panel of system

In this experimental system, the current detection circuit plays an important role, for detecting the DC bus current not only in order to achieve zero initial position detection, but also need real-time detection whether the bus current flow, current protection is an important component part. At present, the current detection methods include sampling resistance, magnetic resistance method and current sensor method. In the case of considering the cost and performance of the system, the relative resistance of sampling methods and the accuracy of the other two a little low, but the corresponding reduced costs, this article uses the resistance sampling method.

Sampling resistor current sensing principle is: the control system on the bus in series between the ground and a little known resistance and then detecting the voltage drop across the resistor, Ohm's law can be calculated according to the current value. After sampling resistor LM358 amplifier to produce a certain amount of voltage, input the microcontroller A / D sampling ports, real-time sampling to complete the current, Fig.3 shows the software running current detection. 


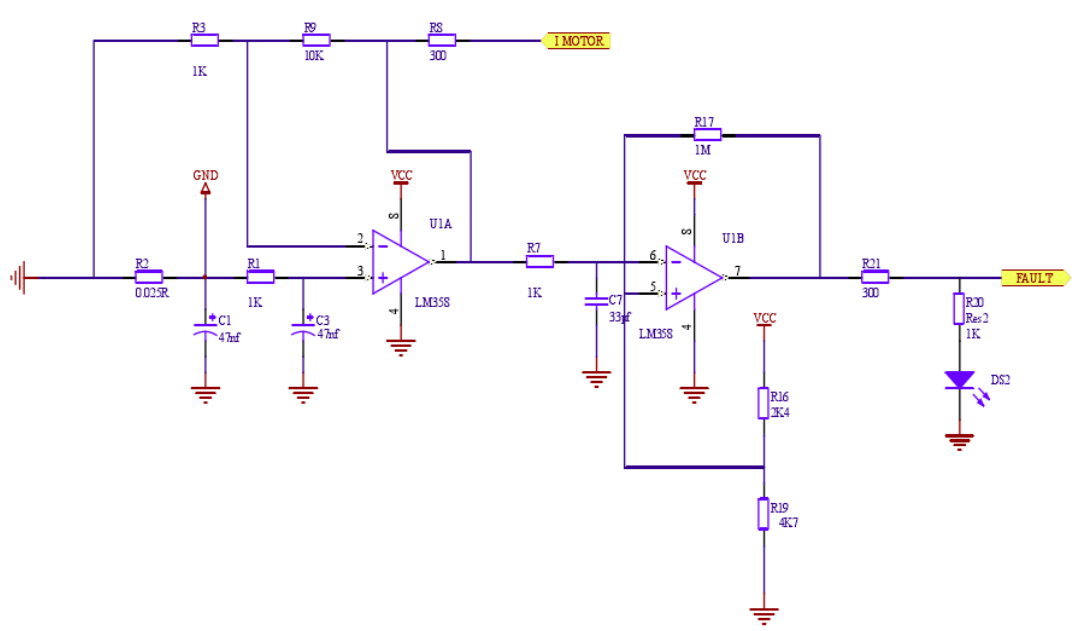

Fig. 3 .The software running current detection

\section{Conclusions}

DC motors with its unique advantages on a wide range of applications. This paper analyzes the microcontroller as a brushless DC motor control system controller, has practical significance of the study and a good market value. This article is designed PIC microcontroller-based DC motor controller has a simple hardware structure, the protection functions, the software uses a modular design for ease of secondary development and so on. Determine the hardware platform and control system, enabling a digital control system. Design software program takes full advantage of the processor's available resources, rational planning code which are special requirements for digital signal filtering to improve the performance of programs to ensure the stability of the program execution to achieve a digital PI algorithm closed-loop speed control and speed, dual-loop control system.

\section{References}

[1] Yang L, Li H, Shen M Y Speed control of BLDCM for industrial sewing machine Based on DSPACE.IEEE 2006 International Conference on Mechatronics and Automation, 2006 (11):2127-2132.

[2] Lin Xu;Yan Jiang;Jian hui Wang;Tong Gao; Xiang yi De, Design of Fuzzy-PID controller based on prediction model and its realization in PLC[J].Control and Decision Conference, 2008. CCDC. Chinese .2008:3808-3811.

[3] YXIA C L, LI Z Q, SHI T N.A control strategy for four-switch three-phase brushless DC motor using single current sensor [J].IEEE Transactons on industrial electronics, Vol. 56, NO. 6, June 2009:2058-2066.

[4] Lin X Q, Zhang H L, Han B. The Design of Fuzzy Control for Linear Motor Based on TMS320F2812.Chinese 2009 Control and Decision Conference,2009(1):5369-5373.

[5] Ren Hai Peng, Liu Ding. A novel digital position servo system using DSP and Fuzzy PID. In: Proceedings of the Fifth International Conference on ICEMS 2011. Shenyang, 2011, 722-725. 